\title{
Chrysin protects human osteoarthritis chondrocytes by inhibiting inflammatory mediator expression via HMGB1 suppression
}

\author{
CHI ZHANG ${ }^{1,2^{*}}$, WEIZHONG YU ${ }^{3 *}$, CHONGBO HUANG $^{3}$, QINGHE DING ${ }^{3}$, CHIZHANG LIANG $^{3}$, \\ $\mathrm{LE} \mathrm{WANG}^{1,2}$, ZHIQI HOU ${ }^{3}$ and ZHIYONG ZHANG ${ }^{2}$ \\ ${ }^{1}$ Orthopedic Department, The Third Affiliated Hospital of Guangzhou Medical University; \\ ${ }^{2}$ Translational Research Centre of Regenerative Medicine and 3D Printing Technologies of Guangzhou Medical University, \\ State Key Laboratory of Respiratory Disease, The Third Affiliated Hospital of Guangzhou Medical University, \\ Guangzhou, Guangdong 510150; ${ }^{3}$ Department of Knee Surgery and Sport Medicine, \\ Guangzhou Orthopedic Hospital, Guangzhou, Guangdong 510045, P.R. China
}

Received September 28, 2017; Accepted September 19, 2018

DOI: $10.3892 / \mathrm{mmr} .2018 .9724$

\begin{abstract}
High-mobility group box chromosomal protein (HMGB-1) contributes to osteoarthritis (OA) by modulating various oxidative, inflammatory and apoptotic signaling pathways. The effect of chrysin $(\mathrm{CH})$, a natural plant flavonoid, and its functional interaction with HMGB-1, was investigated in a chondrocyte model of OA. Human chondrocytes were pre-treated with $\mathrm{CH}$, and then subsequently treated with IL-1 $\beta$ to induce the formation of chondrocytes similar to those found in OA joints. Next, the expression level of HMGB-1 was determined by immunofluorescence and western blot analysis. Additionally, inflammatory factor expression was measured by ELISA, and cell apoptosis was analyzed with flow cytometry. To further explore the effects of $\mathrm{CH}, \mathrm{HMGB}-1$ expression was silenced following $\mathrm{CH}$ treatment with small interfering (si)RNA. The results demonstrated that $\mathrm{CH}$ inhibited cell apoptosis, dose-dependently reduced matrix metalloproteinase (MMP) 13, collagenase and IL-6 expression, and increased collagen $\alpha-1$ (II) chain (COL2A1) expression in human osteoarthritis chondrocytes. These effects of $\mathrm{CH}$ were
\end{abstract}

Correspondence to: Professor Zhiqi Hou, Department of Knee Surgery and Sport Medicine, Guangzhou Orthopedic Hospital, 449 Middle Dongfeng Road, Guangzhou, Guangdong 510045, P.R. China

E-mail: orthopedic_hzq@yeah.net

Dr Zhiyong Zhang, Translational Research Centre of Regenerative Medicine and 3D Printing Technologies of Guangzhou Medical University, State Key Laboratory of Respiratory Disease, The Third Affiliated Hospital of Guangzhou Medical University, 63 Duobao Road, Liwan, Guangzhou, Guangdong 510150, P.R. China

E-mail: mr.zhiyong@gmail.com

*Contributed equally

Key words: chrysin, high-mobility group box chromosomal protein-1, osteoarthritis, human chondrocytes accompanied by decreased HMGB-1 expression. Additionally, the expression of MMP13, collagenase, IL-6 and COL2A1, as well as apoptosis, was significantly reduced by HMGB-1 siRNA. These results demonstrated that HMGB-1 is critical for the protective effect of $\mathrm{CH}$ on human osteoarthritis chondrocytes, including cell apoptosis and inflammatory factor inhibition, which suggests that $\mathrm{CH}$ may have potential therapeutic effect in treating OA by protecting human osteoarthritis chondrocytes via HMGB1 suppression.

\section{Introduction}

As the most common type of human arthritis and musculoskeletal disease, osteoarthritis (OA) is a degenerative and chronic joint disorder caused by the deterioration of hyaline cartilage, accompanied by chondrocyte hypertrophy, angiogenesis, chondrogenesis, and variable degrees of inflammation without systemic effects $(1,2)$. Several important inflammatory factors, including interleukin (IL)-1 $\beta$, tumor necrosis factor $\alpha$, nitric oxide, matrix metalloproteinases (MMPs) and eicosanoids, are actively synthesized during dysfunctional cartilage homeostasis, which result in increased nuclear factor (NF)- $\mathrm{kB}$ and catabolic activity (3-5). Although numerous studies have revealed the contribution of genetic factors to OA, inflammatory factors and altered chondrocyte responses also contribute to OA progression (6-8). The etiology of OA is thought to be influenced by aging, genetics, trauma and obesity. Furthermore, a molecular target for treating OA has yet to be identified $(9,10)$. As a result, the current treatment options predominantly consist of pain management, and no disease-modifying agent to effectively treat OA is currently available.

High-mobility group box chromosomal protein (HMGB-1) is a ubiquitous nuclear DNA-binding protein with a mass of $\sim 27 \mathrm{kDa}$, which contains an amino acid sequence that is highly conserved between rodents and humans (11). Activation of HMGB-1 is typically triggered by necrotic cells, macrophages or other myeloid cells in response to an inflammatory stimulus (12). Previous studies have detected high levels of HMGB-1 in the synovial fluid of patients with 
rheumatoid arthritis and collagen-induced arthritis animal models $(11,13)$. These high HMGB-1 levels were shown to induce MMP and cytokine production, as well as angiogenesis, by enhancing oxidative stress in vitro (14). In addition, in vitro studies suggest that the chondrocyte hypertrophy and increased synthesis of type $X$ collagen caused by OA may be driven by the HMGB-1 receptor, the receptor for advanced glycated end-products (RAGE) (15). As an important pro-inflammatory mediator, HMGB1, along with its receptor, have been associated with the onset and progression of cancers and arthritis (16,17); however, a limited number of studies have investigated HMGB1 and its various downstream genes as possible therapeutic targets in OA $(18,19)$.

Chrysin ( $\mathrm{CH}$; 5,7-dihydroxyflavone), an important natural plant flavonoid, has been demonstrated to exert antioxidative, anti-allergic, anti-inflammatory, antifibrotic and anti-apoptotic effects in the central nervous and immune systems $(20,21)$. However, few studies have investigated the potential use of $\mathrm{CH}$ for treating OA, despite the recently demonstrated ability of $\mathrm{CH}$ to inhibit inflammatory factor stimulation, and to produce therapeutic effects in human OA chondrocytes in vitro (22).

Results of previous studies have suggested an upregulation of HMGB-1 and inflammatory cytokine expression, including IL- 6 or IL-8, in OA cartilage $(23,24)$. Accordingly, the present study was designed to determine whether treatment with $\mathrm{CH}$ improved the characteristics of human OA chondrocytes by activating HMGB1, and thereby altering the production of inflammatory factors. The alterations in cellular function and inflammatory factors which occurred following HMGB-1 silencing were also examined. To the best our knowledge, this is the first study to evaluate the protective effects of $\mathrm{CH}$, and to investigate the involvement of HMGB-1 in OA in vitro. The results of the present study may assist in the discovery of novel treatments for OA.

\section{Materials and methods}

Cell culture and treatment. Human chondrocytes (HC-a) were obtained from Shanghai CAFA Biological Technology (Shanghai, China). Cells were cultured for $24 \mathrm{~h}$ in high glucose-Dulbecco's modified Eagle's medium (DMEM) supplemented with 10\% fetal bovine serum (FBS) (both from HyClone; GE Healthcare Life Sciences, Logan, UT, USA) and $1 \%$ penicillin/streptomycin (Corning Incorporation, Corning, $\mathrm{NY}$, USA) in a humidified atmosphere with $5 \% \mathrm{CO}_{2}$ at $37^{\circ} \mathrm{C}$.

Following culture, the cells were diluted to single cell suspensions and seeded into 6 -well plates $\left(1 \times 10^{4}\right.$ cells/well). Next, an OA model was induced by incubating the cells with IL-1 $\beta(200 \mu \mathrm{M})$ for $24 \mathrm{~h}$ at $37^{\circ} \mathrm{C}$. For $\mathrm{CH}$ treatment, the cells were incubated with $0,0.4$, and $4 \mu \mathrm{M} \mathrm{CH}$ (cat. no. $\mathrm{C} 80105$, Sigma-Aldrich; Merck KGaA, Darmstadt, Germany) for $24 \mathrm{~h}$ at $37^{\circ} \mathrm{C}$, respectively. For transfection, the $50 \mathrm{nM}$ HMGB1 siRNA (siRNA) and $50 \mathrm{nM}$ negative control (NC) oligonucleotides were synthesized from Shanghai GenePharma, Co., Ltd. (Shanghai, China). The sequences were si-HMGB1, 5'-CCC GUUAUGAAAGAGAAAUUU-3' (sense), 5'-AUUUCUCUU UCAUAAUGGGUU-3' (antisense); si-NC, 5'-UUCGUCUGU ACUCCACAUATT-3' (sense), 5'-GAUGUCUUCUACAGU CCGATT-3' (antisense). The cells were transfected with si-NC or si-HMGB1 by using Lipofectamine ${ }^{\circledR} 2000$ (Invitrogen;
Thermo Fisher Scientific, Inc., Waltham, MA, USA) for $48 \mathrm{~h}$ at $37^{\circ} \mathrm{C}$ following the manufacturer's protocols. Non-treated cells were used as a blank group.

Thus, the initial experimental groups were as follows: i) Blank (non-treated cells); ii) OA model (treated with $200 \mu \mathrm{M}$ IL-1 $\beta$ ); and iii) $\mathrm{CH}$ (treated with $200 \mu \mathrm{M}$ IL-1 $\beta$ and the indicated concentration of $\mathrm{CH}$ ). The transfection experimental groups were: i) Blank (non-treated cells); ii) OA model (treated with $200 \mu \mathrm{M}$ IL-1 $\beta$ ); iii) NC (treated with $200 \mu \mathrm{M}$ IL-1 $\beta$, si-NC); iv) siRNA (treated with $200 \mu \mathrm{M}$ IL-1 $\beta$ and si-HMGB1); and v) $\mathrm{CH}+\operatorname{siRNA}$ (treated with $200 \mu \mathrm{M}$ IL-1 $\beta$, si-HMGB1 and CH).

Apoptosis assay. Apoptotic cells were quantified using an Annexin V-fluorescein isothiocyanate (FITC)/propidium iodide (PI) apoptosis detection kit (Merck KGaA). Cells were collected by $0.25 \%$ trypsin digestion, washed with PBS and re-suspended in $200 \mu \mathrm{l}$ binding buffer containing $5 \mu \mathrm{l}$ Annexin $\mathrm{V}(10 \mu \mathrm{g} / \mathrm{ml})$ in DMEM with FBS at $37^{\circ} \mathrm{C}$ for $10 \mathrm{~min}$ in the dark. The cells were subsequently incubated with $10 \mu \mathrm{l} \mathrm{PI}(20 \mu \mathrm{g} / \mathrm{ml})$ for $15 \mathrm{~min}$ at room temperature and analyzed with the EPICS ${ }^{\circledR}$ XL $^{\mathrm{TM}}$ flow cytometer (Beckman Coulter, Inc., Brea, CA, USA). Data acquisition and analyses were performed using CellQuest ${ }^{\mathrm{TM}}$ software version 5.1 (BD Biosciences, Franklin Lakes, NJ, USA). Early and late apoptotic cells were detected in the lower and upper right quadrants of the flow cytometry plots presented late apoptosis, and lower right represented early apoptosis. The percentage of apoptotic cells was presented for both early and late apoptotic cells.

Immunofluorescence staining. Cells were plated onto coverslips and incubated in RPMI-1640 medium (cat. no. 11875-093; Gibco; Thermo Fisher Scientific, Inc.) containing $10 \% \mathrm{FBS}$ for $24 \mathrm{~h}$ at $37^{\circ} \mathrm{C}$. Following treatment, cells were fixed with $4 \%$ paraformaldehyde for $20 \mathrm{~min}$ at $4^{\circ} \mathrm{C}$, incubated in $0.3 \%$ Triton X-100-PBS for $10 \mathrm{~min}$ at room temperature and subsequently blocked with $5 \%$ goat serum at $37^{\circ} \mathrm{C}$ for $30 \mathrm{~min}$. The cells were incubated with anti-human HMGB-1 (1:2,000; cat no. M-1702-100; Biosensis Pty Ltd., Thebarton, Australia) at $4{ }^{\circ} \mathrm{C}$ overnight, followed by incubation with goat anti-human immunoglobulin $\mathrm{G}$ conjugated to Cy3 (1:400; Jackson ImmunoResearch Laboratories, Inc., West Grove, PA, USA) at $37^{\circ} \mathrm{C}$ for $1 \mathrm{~h}$. Nuclei were counter stained with DAPI (1:1,000; Sigma-Aldrich; Merck KGaA) for $5 \mathrm{~min}$ at room temperature. Images were obtained using an inverted fluorescence microscope (Olympus Corporation, Tokyo, Japan) at x400 magnification.

Protein isolation and western blot analysis. The total protein was extracted from cells by incubation with lysis buffer $(12.5 \mathrm{ml}$ Tris HCL, $2 \mathrm{~g} \mathrm{SDS}, 10 \mathrm{ml}$ glycerol and $67.5 \mathrm{ml}$ distilled water). Nuclear protein was extracted with NE-PER Nuclear and Cytoplasmic Extraction Reagents (Thermo Fisher Scientific, Inc., Waltham, MA, USA) according to the manufacturer's protocols. The concentrations were measured by the protein assay kit (Qcbio Science Technologies Co., Ltd., Shanghai, China). The protein $(30 \mu \mathrm{g})$ were separated by electrophoresis on Novex ${ }^{\circledR} 4-20 \%$ Tris-Glycine 12-well polyacrylamide gradient gels (Invitrogen; Thermo Fisher Scientific, Inc.). Subsequently, the separated proteins were transferred onto a nitrocellulose membrane by using a Protean Mini Cell system 
(Bio-Rad Laboratories, Inc., Hercules, CA, USA). The gel was blocked with $5 \%$ non-fat milk in Tris-buffered saline with $0.1 \%$ Tween-20 (TBST; Merck KGaA) for $2 \mathrm{~h}$ at room temperature. The membrane was incubated with anti-human HMGB-1 (1:10,000, ab77302; Abcam, Cambridge, UK), anti-GAPDH as a loading control (1:2,000, sc-47724; Santa Cruz Biotechnology, Inc., Dallas, CA, USA), and lamin B (1:2,000, ab122919; Abcam) overnight at $4^{\circ} \mathrm{C}$, followed by blotting with horseradish peroxidase-conjugated secondary antibodies (1:2,000, anti-mouse, cat. no. SC-2005 and anti-rabbit, cat. no. SC-2004) for $1 \mathrm{~h}$ at room temperature; following which, it was washed again with TBST. Finally, the blots were analyzed by the enhanced chemiluminescence (ECL) substrate kit an ECL system (both from GE Healthcare, Chicago, IL, USA).

ELISA. The concentrations of MMP13 (1:5,000, ab9128), collagenase (1:5,000, ab182881), IL-6 (1:5,000, ab7737) and collagen $\alpha-1$ (II) chain (COL2A1, 1:5,000, ab34712) (all from Abcam) were quantified with commercial human ELISA kits (Elabscience, Wuhan, China) according to the manufacturer's protocols. All samples were assayed in duplicate. The mean concentration was determined for each sample. Stop solution was then added to each well, and its optical density at $450 \mathrm{~nm}\left(\mathrm{OD}_{450}\right)$ was immediately measured on an Infinite M200 microtiter plate reader (Tecan Group, Ltd., Maennedorf, Switzerland).

Statistical analysis. All statistical analyses were performed using SPSS 19.0 (IBM Corp., Armonk, NY, USA). Data were presented as the mean \pm standard deviation. Student's t-test was used to analyze differences between two groups, and one-way analysis of variance followed by Tukey's post-hoc test was used to determine the significance of differences among multiple groups. $\mathrm{P}<0.05$ was considered to indicate a statistically significant difference. All experiments were independently repeated three times.

\section{Results}

HMGB-1 expression in the human chondrocyte OA model. To validate the OA cell model used in this study, HMGB-1 expression was detected in human chondrocytes following pre-treatment with IL-1 $\beta$, followed by treatment with $\mathrm{CH}$. The results demonstrated that the HMGB1 expression levels were increased in response to IL-1 treatment, but was notably decreased in the $\mathrm{CH}$ treated groups, compared with the $\mathrm{OA}$ group (Fig. 1A). The results from immunofluorescence assays revealed that the increase in HMGB-1 expression in response to IL-1 $\beta$, followed by a dose-dependent decrease in HMGB-1 expression in response to $\mathrm{CH}$ (Fig. 1B).

$\mathrm{CH}$ treatment alters the expression of inflammatory mediators and reduces apoptosis. The results of the ELISAs are presented in Fig. 2. Compared with the blank group, the levels of MMP13, collagenase and IL-6 were significantly increased in the OA model group; however, that of COL2A1 were significantly decreased. Compared with the OA model group, the levels of MMP13, collagenase and IL-6 were significantly decreased in the $\mathrm{CH}$ treatment groups; however that of COL2A1 were significantly increased in the $\mathrm{CH}(4 \mu \mathrm{M})$
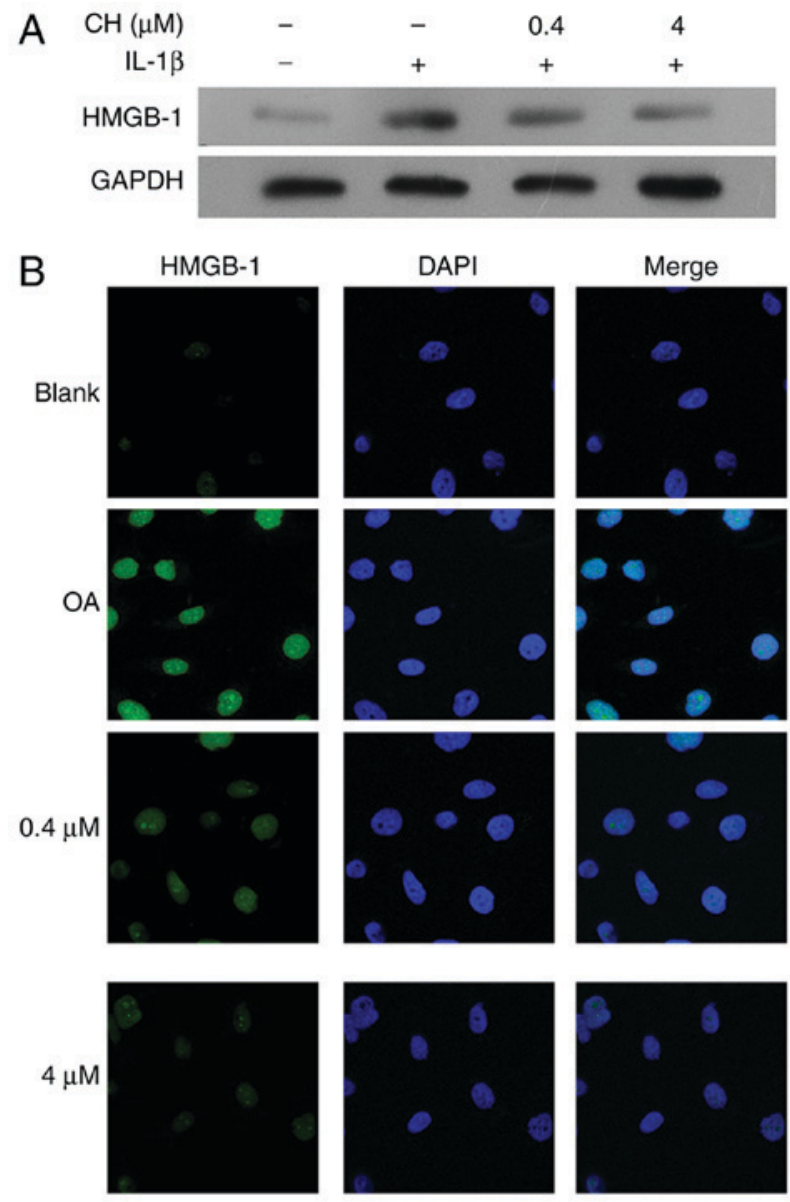

Figure 1. Differential expression of HMGB-1 in human chondrocytes in response to IL-1 $\beta$ and $\mathrm{CH}$ treatments. (A) HMGB-1 expression was detected by western blotting. (B) HMGB-1 expression and localization was investigated by immunofluorescence and observed with a laser scanning confocal microscope. Regions with HMGB-1 are green; cell nuclei were stained with DAPI. Magnification, $\mathrm{x} 400$, scale bar=20 $\mu \mathrm{m} . \mathrm{CH}$, chrysin; IL-1 $\beta$, interleukin-1 $\beta$; HMGB-1, high-mobility group box chromosomal protein-1; OA, osteoarthritis model.

group. The results suggested that $\mathrm{CH}$ treatment inhibited the levels of inflammatory mediators in IL- $1 \beta$-induced $\mathrm{HC}$-a cells (P<0.01; Fig. 2).

Additionally, cell apoptosis was analyzed by flow cytometry following treatment with IL- $1 \beta$ and $\mathrm{CH}$. The results demonstrated that the number of apoptotic cells was significantly increased in OA model group compared with the blank group, while the number of apoptotic cells was significantly decreased in the $\mathrm{CH}$ treatment groups compared with the $\mathrm{OA}$ model group $(\mathrm{P}<0.01$; Fig. 3). The results indicated that $\mathrm{CH}$ treatment suppressed the apoptotic ability of IL- $1 \beta$-induced HC-a cells.

Validation of HMGB-1 knockdown in the human chondrocyte OA model. Following treatment with IL-1 $\beta$ for $24 \mathrm{~h}$, OA model chondrocytes were transfected with si-HMGB-1 and/or treated with $\mathrm{CH}(4 \mu \mathrm{M})$. As presented in Fig. 4A and B, HMGB-1 expression in the siRNA-transfected cells was significantly inhibited when compared with HMGB-1 expression in cells transfected with the si-HMGB-1-NC control (NC group). Immunofluorescence analyses were performed to demonstrate that in the nucleus, HMGB-1 silenced cells 
A

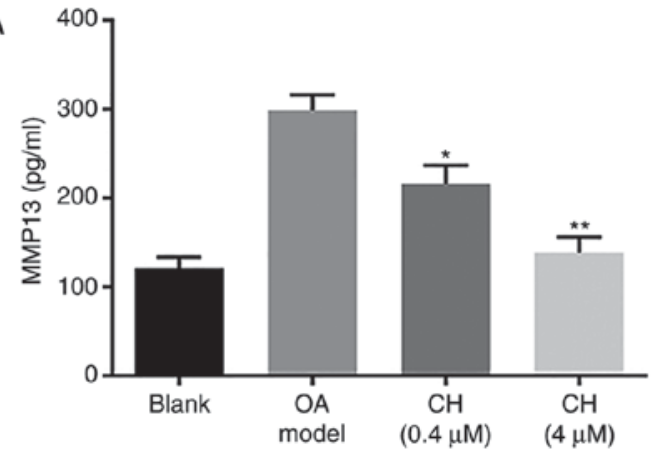

C

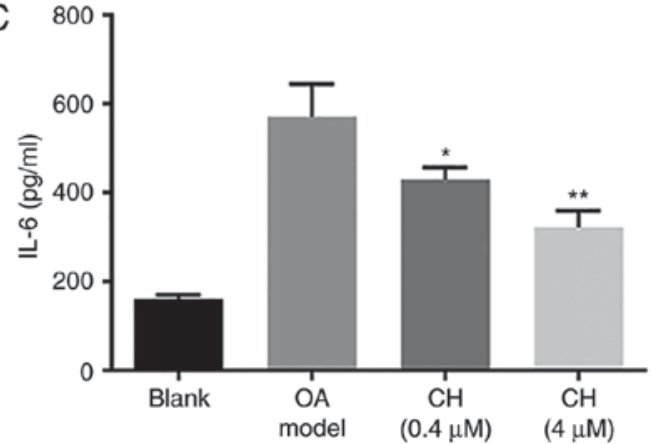

B

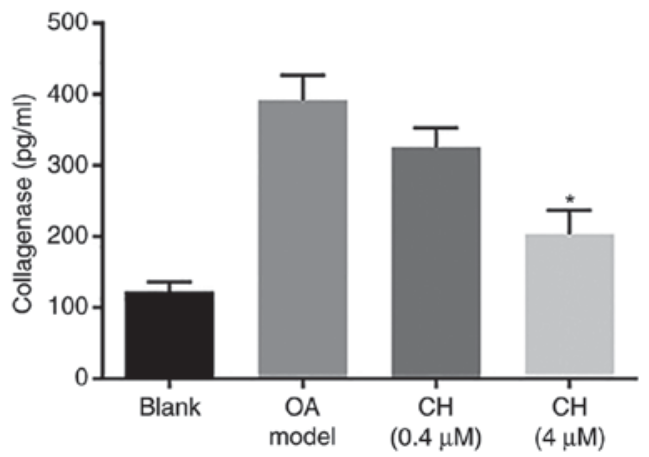

D

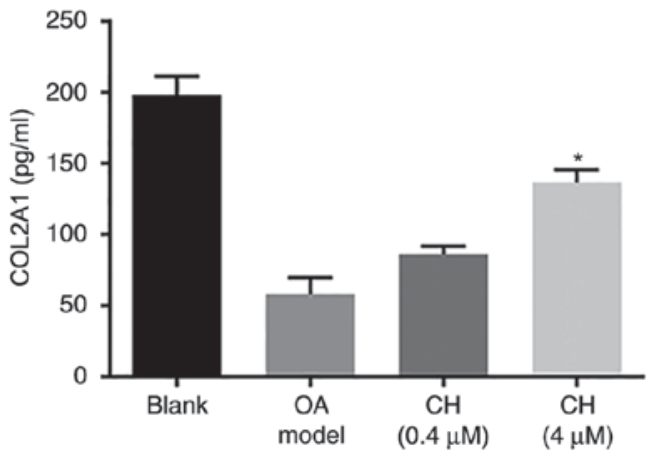

Figure 2. MMP13, collagenase, IL-6 and COL2A1 expression levels. (A) MMP13, (B) collagenase, (C) IL-6 and (D) COL2A1 expression in medium $24 \mathrm{~h}$ after IL-1 $\beta$ and $\mathrm{CH}$ treatments. Expression levels were detected by ELISA. Data are presented as the mean \pm standard deviation. ${ }^{*} \mathrm{P}<0.05,{ }^{* *} \mathrm{P}<0.01$, vs. OA model group. $\mathrm{CH}$, chrysin; OA, osteoarthritis model; IL-6, interleukin-6; MMP13, matrix metalloproteinase 13; COL2A1, collagen $\alpha-1$ (II) chain.
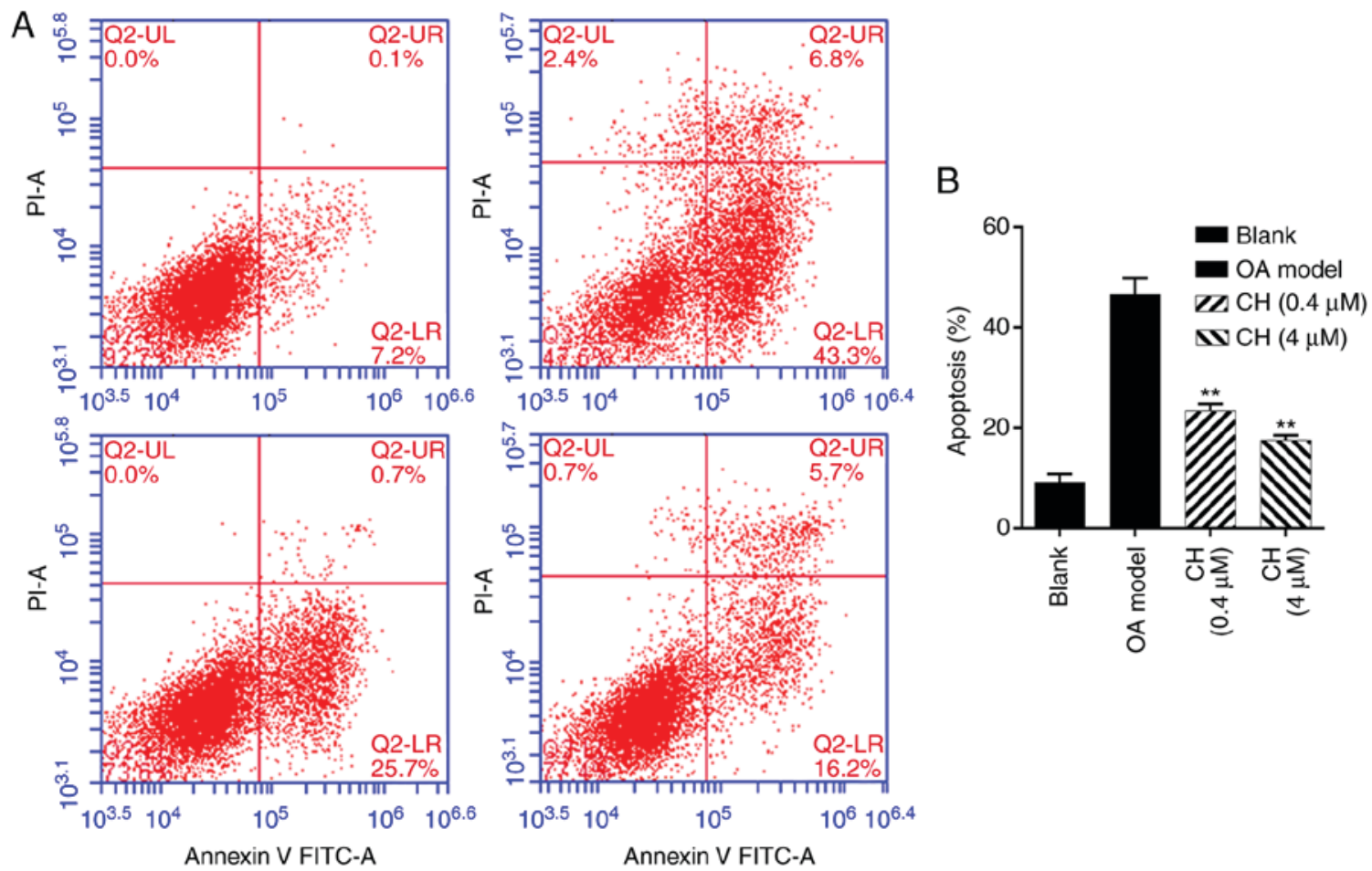

Figure 3. CH reduced the number of apoptotic cells in aliquots of IL-1 $\beta$-treated cells. (A) Flow cytometry results. (B) The percentage of apoptotic cells was statistically analyzed. Data are presented as the mean \pm standard deviation. Experiments were independently repeated three times. ${ }^{* *} \mathrm{P}<0.01 \mathrm{vs}$. the model group. IL-1 $\beta$, interleukin-1 $\beta$; $\mathrm{CH}$, chrysin; OA, osteoarthritis model; FITC, fluorescein isothiocyanate; PI, propidium iodide.

emitted less florescence compared with cells in the NC group (Fig. 4B and C). The results suggested that silencing of HMGB1 and $\mathrm{CH}$ treatment downregulated the expression levels of the total and nuclear HMGB-1 protein in OA model cells.
CH and siRNA cotreatment further reduces apoptosis. ELISA assays was performed to the detect the concentrations of MMP13, collagenase, IL-6 and COL2A1. The results proved that compared with the OA group, the expression of MMP13, 


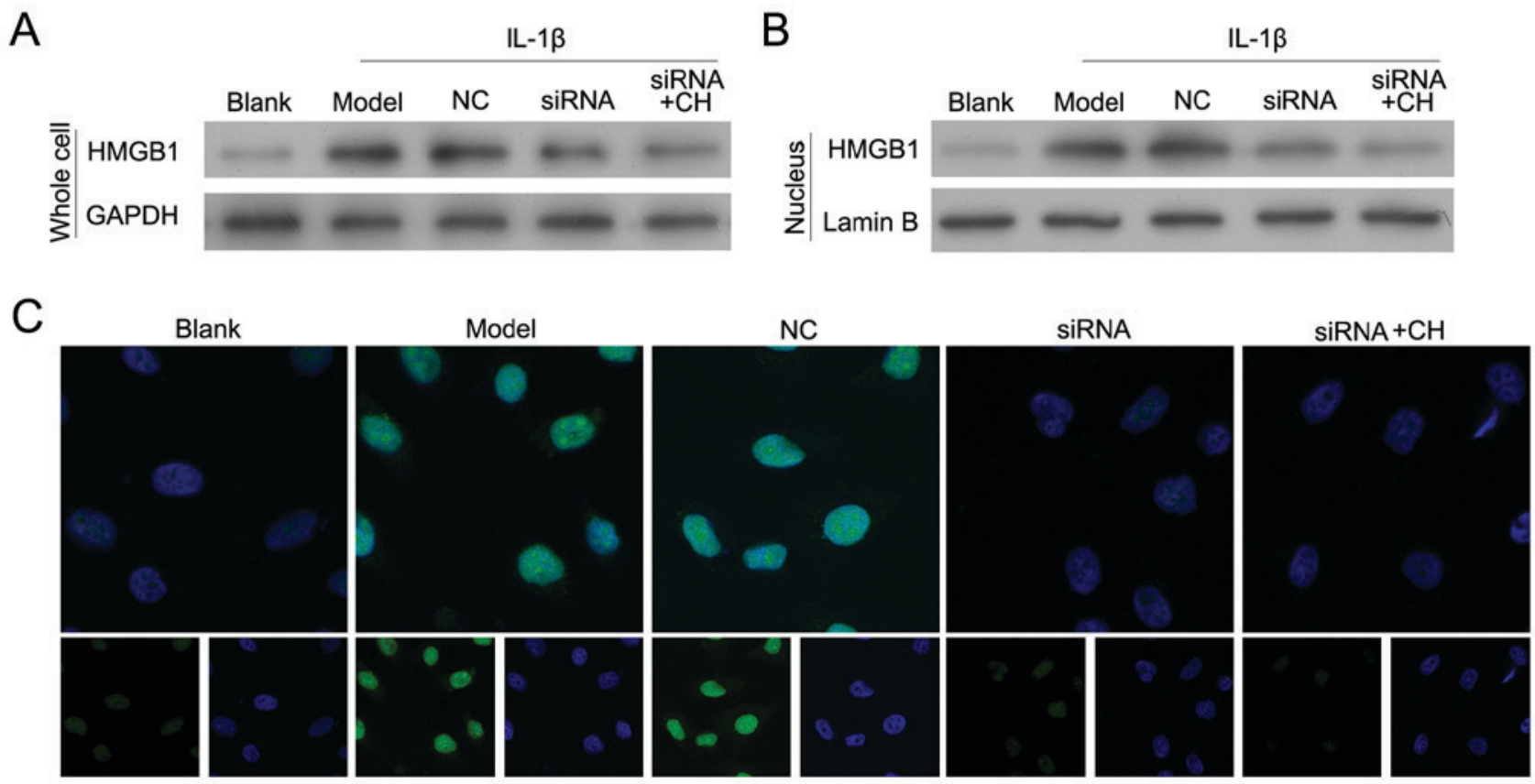

Figure 4. Validation of HMGB-1 levels in OA human chondrocyte model cells. Following CH treatment, HMGB-1 was silenced by HMGB-1-specific siRNA or transfected with NC siRNA. (A) HMGB1 expression in whole cells from each group. (B) HMGB1 expression in the nucleus of each group. (C) HMGB1 immunofluorescence was observed in cells under a laser scanning confocal microscope. Regions with HMGB-1 are green; cell nuclei were stained with DAPI. Magnification, $\mathrm{x} 400$, scale bar=20 $\mu \mathrm{m}$. CH, chrysin; HMGB-1, high-mobility group box chromosomal protein-1; siRNA, small interfering RNA; NC, negative control.
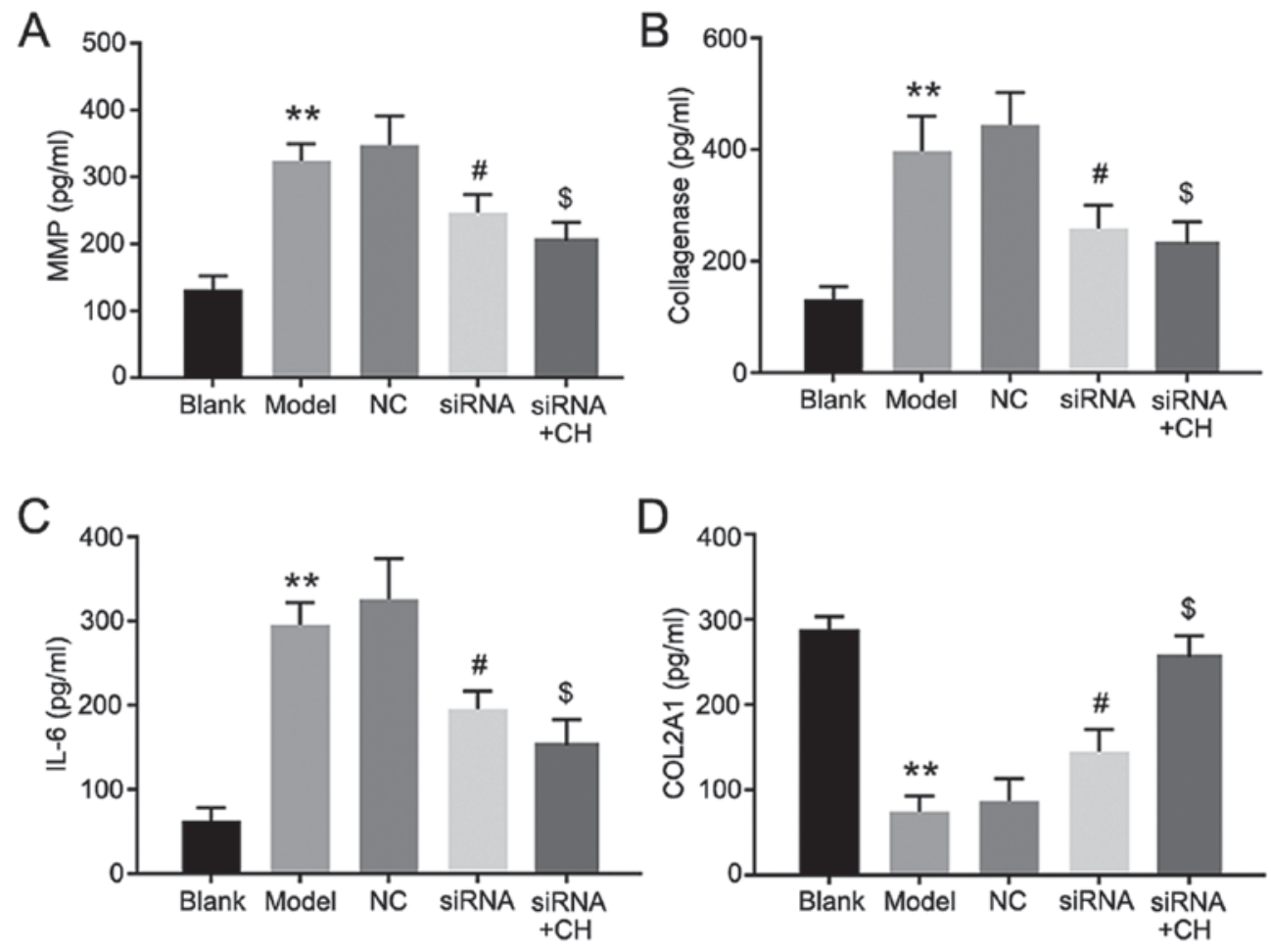

Figure 5.MMP13, collagenase, IL-6, and COL2A1 expressions levels following the silencing of HMGB-1. (A) MMP13, (B) collagenase, (C) IL-6 and (D) COL2A1 expression in medium. Data are presented as the mean \pm standard deviation. Experiments were independently repeated three times. ${ }^{* *} \mathrm{P}<0.01$ vs. blank group; ${ }^{\#} \mathrm{P}<0.05$ vs. NC group; ${ }^{\text {P}}<0.05$ vs. siRNA group. $\mathrm{CH}$, chrysin; IL-6, interleukin-6; MMP13, matrix metalloproteinase 13; COL2A1, collagen $\alpha-1$ (II) chain; siRNA, small interfering RNA; NC, negative control.

collagenase and IL-6 was decreased following HMGB-1 knockdown, while COL2A1 expression was increased. Additionally, MMP13, collagenase and IL-6 expression was further reduced in $\mathrm{CH}$ and si-HMGB-1 treated cells, compared with cells transfected with si-HMGB-1 alone. Furthermore, COL2A1 expression was significantly increased in the $\mathrm{CH}$ and si-HMGB treatment group, compared with the siRNA group $(\mathrm{P}<0.05$, $\mathrm{P}<0.01$; Fig. 5). Flow cytometry revealed that the number of 

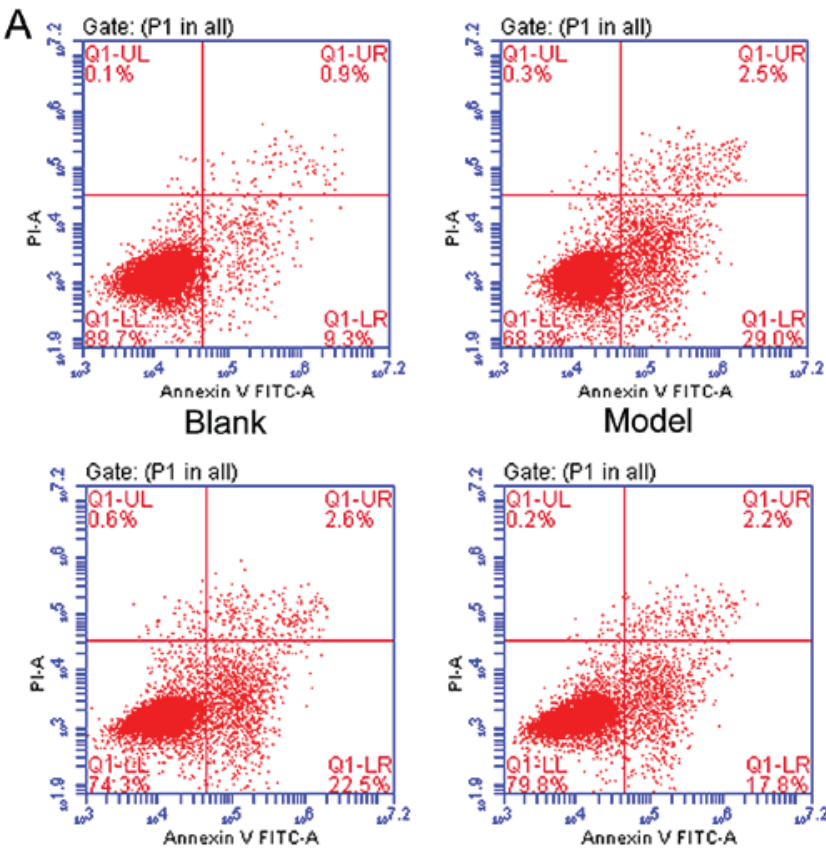

NC

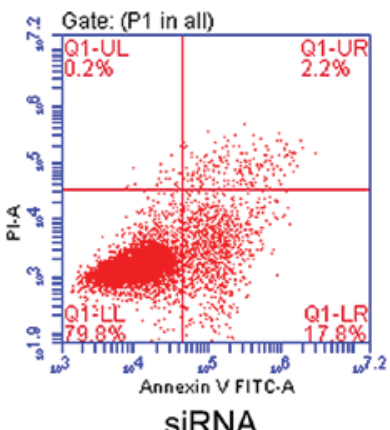

B

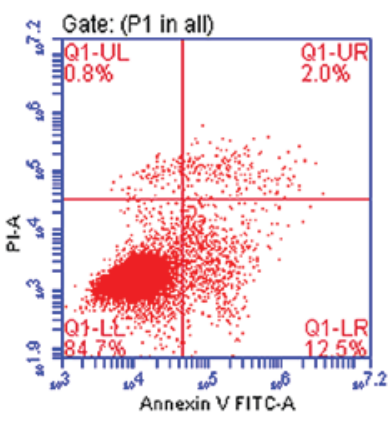

SiRNA+CH

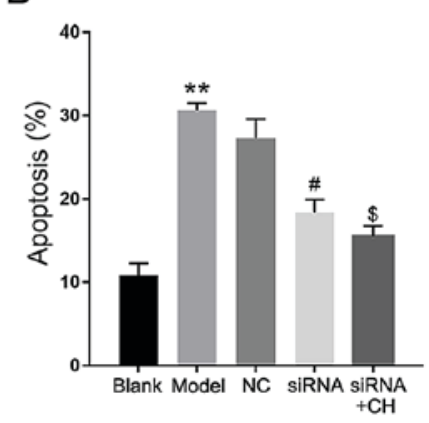

Figure 6. Apoptosis in each cell group following knockdown of HMGB-1. (A) Flow cytometry results. (B) The percentage of apoptotic cells was determined. ${ }^{* *} \mathrm{P}<0.01$ vs. blank group; ${ }^{*} \mathrm{P}<0.05$ vs. NC group; ${ }^{\$} \mathrm{P}<0.05$ vs. siRNA group. $\mathrm{CH}$, chrysin; HMGB-1, high-mobility group box chromosomal protein-1; siRNA, small interfering RNA; NC, negative control.

apoptotic cells was significantly increased in OA model group compared with the blank group. Silencing of HMGB1 significantly inhibited the apoptotic potential of IL-1 $\beta$-induced $\mathrm{HC}$-a cells compared with the NC group; treatment with $\mathrm{CH}$ enhanced the inhibition mediated by HMGB1 siRNA compared with the silencing group $(\mathrm{P}<0.05, \mathrm{P}<0.01$; Fig. 6$)$.

\section{Discussion}

Complementary and alternative medical techniques, including certain traditional Chinese medicines (TCMs), including paeonol (25), isofraxidin (26) and Jingui external lotion (27), have been widely used for treating OA for centuries; these are primarily thought to produce chondroprotective effects and even repair cartilage $(28,29)$. Consequently, there is growing interest in exploring TCMs as potential drugs that may aid in reducing inflammation, protecting cartilage against damage, improving joint function and restoring a patient's activity level (30). The present study was designed to evaluate whether $\mathrm{CH}$ may be effective against $\mathrm{OA}$. This was investigated using an in vitro cartilage cell model to examine if $\mathrm{CH}$ exerted a positive effect on cartilage health through targeting HMGB-1.

As previously reported, HMGB-1 is one of several nuclear DNA-binding proteins that may be passively released in response to an inflammatory stimulus resulting from an OA injury (19). Abundant evidence indicates that certain complexes (cluster of differentiation 24, siglec-10 and tumor-infiltrating dentric cells) induce innate immune responses, and the production of inflammatory mediators is induced by the binding of HMGB1 to bacterial products $(31,32)$. Extracellular HMGB1 has been reported to induce cell proliferation, migration and differentiation by interacting with RAGEs and toll-like receptors (TLRs), including TLR-2 and TLR-4 $(33,34)$. Interactions between HMGB1 and phosphatidylserine on the cell surface inhibit the phagocytosis of apoptotic neutrophils by macrophages, which may lead to the activation of monocytes, macrophages and dendritic cells, which prevents the resolution of inflammation $(2,35,36)$.

Chondrocyte apoptosis is a typical occurrence during OA progression. In response to structural changes in the cartilage matrix, chondrocytes serve a critical role in recreating the anabolic-catabolic balance required for tissue function and matrix maintenance. Therefore, inhibiting chondrocyte apoptosis while promoting the maintenance of healthy chondrocytes represents a potential strategy for preventing cartilage degeneration $(37,38)$. As TCMs are increasingly being used to treat OA (39), scientists have suggested that certain TCMs, particularly those used in combination formulas, may produce therapeutic effects in a synergistic manner. For example, XuanHuSuo powder (XHSP), a conventional herbal formulation developed in China, has been extensively used in OA treatment (40). XHSP has shown reasonable efficacy as an anti-apoptotic and anti-inflammatory agent when applied following stimulation with a cytokine (IL-1 $\beta$ ) or estrogen (40). As an active component of various Chinese herbs, berberine chloride has been demonstrated to benefit matrix synthesis and cell survival in IL-1 $\beta$-stimulated chondrocytes, and displays great therapeutic potential as a promoter of cartilage repair in rat OA models (41). However, there is no effective way to target and promote cartilage protection at present. To the best of our knowledge, the present study was the first to demonstrate that the herbal extract $\mathrm{CH}$ may inhibit chondrocyte apoptosis by targeting HMGB-1.

Taken together, the data indicated that $\mathrm{CH}$ ameliorated $\mathrm{OA}$ in vitro. It does this, at least partially, by inhibiting various processes mediated by HMGB-1, including chondrocyte apoptosis, cellular inflammatory responses and inflammatory cytokine generation. Further studies in animal models are required to evaluate the safety and efficacy of this herbal extract.

\section{Acknowledgements}

Not applicable.

\section{Funding}

This research was supported by the National Natural Science Foundation of China (grant no. 81702196) and the Province Natural Science Fund of Guangdong (grant no. 2017A030313137). 


\section{Availability of data and materials}

All data generated or analyzed during this study are included in this published article.

\section{Authors' contributions}

$\mathrm{CZ}, \mathrm{WZY}$ and ZQH designed the experiments. $\mathrm{CZ}, \mathrm{CBH}$ and QHD performed most of the experiments. CZ, CHL, LW and ZYZ collected and analyzed the data. CZ drafted the manuscript. ZYZ provided the administrative support.

\section{Ethics approval and consent to participate}

Not applicable.

\section{Patient consent for publication}

Not applicable.

\section{Competing interests}

The authors declared that they have no competing interests.

\section{References}

1. Erggelet C, Kreuz PC, Mrosek EH, Schagemann JC, Lahm A, Ducommun PP and Ossendorf C: Autologous chondrocyte implantation versus ACI using 3D-bioresorbable graft for the treatment of large full-thickness cartilage lesions of the knee. Arch Orthop Trauma Surg 130: 957-964, 2010.

2. Ge X, Shi R and Ma X: The secreted protein WNT5A regulates condylar chondrocyte proliferation, hypertrophy and migration. Arch Oral Biol 82: 171-179, 2017.

3. Liao S, Zhou K, Li D, Xie X, Jun F and Wang J: Schisantherin A suppresses interleukin-1 $\beta$-induced inflammation in human chondrocytes via inhibition of NF- $\kappa \mathrm{B}$ and MAPKs activation. Eur J Pharmacol 780: 65-70, 2016.

4. Martinez SE, Chen Y, Ho EA, Martinez SA and Davies NM: Pharmacological effects of a C-phycocyanin-based multicomponent nutraceutical in an in-vitro canine chondrocyte model of osteoarthritis. Can J Vet Res 79: 241-249, 2015.

5. Wang HZ, Jin Y, Wang P, Han C, Wang ZP and Dong MY: Alteration of serum endocan in normal pregnancy and preeclampsia. Clin Exp Obstet Gynecol 44: 419-422, 2017.

6. Xu L, Peng Q, Xuan W, Feng X, Kong X, Zhang M, Tan W, Xue $\mathrm{M}$ and Wang F: Interleukin-29 enhances synovial inflammation and cartilage degradation in osteoarthritis. Mediators Inflamm 2016: $9631510,2016$.

7. Liu-Bryan R and Terkeltaub R: Emerging regulators of the inflammatory process in osteoarthritis. Nat Rev Rheumatol 11: 35-44, 2015

8. Wojdasiewicz P, Poniatowski ŁA and Szukiewicz D: The role of inflammatory and anti-inflammatory cytokines in the pathogenesis of osteoarthritis. Mediators Inflamm 2014: 561459, 2014.

9. Bruyère $\mathrm{O}$, Cooper $\mathrm{C}$, Arden N, Branco J, Brandi ML, HerreroBeaumont G, Berenbaum F, Dennison E, Devogelaer JP, Hochberg M, et al: Can we identify patients with high risk of osteoarthritis progression who will respond to treatment? A focus on epidemiology and phenotype of osteoarthritis. Drugs Aging 32: 179-187, 2015.

10. Kimura T: Progress of research in osteoarthritis. An overview of the recent knowledge on osteoarthritis: Pathogenesis, evaluation and therapies. Clin Calcium 19: 1565-1571, 2009 (In Japanese).

11. Taniguchi N, Kawahara K, Yone K, Hashiguchi T, Yamakuchi M, Goto M, Inoue K, Yamada S, Ijiri K, Matsunaga S, et al: High mobility group box chromosomal protein-1 plays a role in the pathogenesis of rheumatoid arthritis as a novel cytokine. Arthritis Rheum 48: 971-981, 2003.
12. Sunahori K, Yamamura M, Yamana J, Takasugi K, Kawashima M and Makino H: Increased expression of receptor for advanced glycation end products by synovial tissue macrophages in rheumatoid arthritis. Arthritis Rheum 54: 97-104, 2006.

13. Kokkola R, Li J, Sundberg E, Aveberger AC, Palmblad K, Yang H, Tracey KJ, Andersson U and Harris HE: Successful treatment of collagen-induced arthritis in mice and rats by targeting extracellular high mobility group box chromosomal protein-1 activity. Arthritis Rheum 48: 2052-2058, 2003.

14. Hamada T, Torikai M,Kuwazuru A, Tanaka M,Horai N,Fukuda T, Yamada S, Nagayama S, Hashiguchi K, Sunahara N, et al: Extracellular high mobility group box chromosomal protein-1 is a coupling factor for hypoxia and inflammation in arthritis. Arthritis Rheum 58: 2675-2685, 2008.

15. Chen YJ, Sheu ML, Tsai KS, Yang RS and Liu SH: Advanced glycation end products induce peroxisome proliferator-activated receptor $\gamma$ down-regulation-related inflammatory signals in human chondrocytes via Toll-like receptor-4 and receptor for advanced glycation end products. PLoS One 8: e66611, 2013.

16. Sims GP, Rowe DC, Rietdijk ST, Herbst R and Coyle AJ: HMGB1 and RAGE in inflammation and cancer. Annu Rev Immunol 28: 367-388, 2010.

17. Wang WJ, Yin SJ and Rong RQ: PKR and HMGB1 expression and function in rheumatoid arthritis. Genet Mol Res 14: 17864-17870, 2015.

18. Qin Y, Chen Y, Wang W, Wang Z, Tang G, Zhang P, He Z, Liu Y, Dai SM and Shen Q: HMGB1-LPS complex promotes transformation of osteoarthritis synovial fibroblasts to a rheumatoid arthritis synovial fibroblast-like phenotype. Cell Death Dis 5: e1077, 2014.

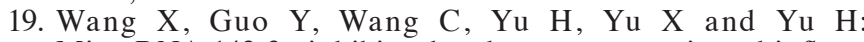
MicroRNA-142-3p inhibits chondrocyte apoptosis and inflammation in osteoarthritis by targeting HMGB1. Inflammation 39: 1718-1728, 2016.

20. Ahad A, Ganai AA, Mujeeb M and Siddiqui WA: Chrysin, an anti-inflammatory molecule, abrogates renal dysfunction in type 2 diabetic rats. Toxicol Appl Pharmacol 279: 1-7, 2014.

21. Khan R, Khan AQ, Qamar W, Lateef A, Ali F, Rehman MU, Tahir M, Sharma S and Sultana S: Chrysin abrogates cisplatin-induced oxidative stress, p53 expression, goblet cell disintegration and apoptotic responses in the jejunum of Wistar rats. Br J Nutr 108: 1574-1585, 2012

22. Zheng W, Tao Z, Cai L, Chen C, Zhang C, Wang Q, Ying X, $\mathrm{Hu} \mathrm{W}$ and Chen $\mathrm{H}$ : Chrysin attenuates IL-1 $\beta$-induced expression of inflammatory mediators by suppressing NF- $\kappa \mathrm{B}$ in human osteoarthritis chondrocytes. Inflammation 40: 1143-1154, 2017.

23. Magna M and Pisetsky DS: The role of HMGB1 in the pathogenesis of inflammatory and autoimmune diseases. Mol Med 20: 138-146, 2014.

24. Xu M, Zhou GM, Wang LH, Zhu L, Liu JM, Wang XD, Li HT and Chen L: Inhibiting high-mobility group box 1 (HMGB1) attenuates inflammatory cytokine expression and neurological deficit in ischemic brain injury following cardiac arrest in rats. Inflammation 39: 1594-1602, 2016.

25. Liu M, Zhong S, Kong R, Shao H, Wang C, Piao H, Lv W, Chu X and Zhao Y: Paeonol alleviates interleukin-1 $\beta$-induced inflammatory responses in chondrocytes during osteoarthritis. Biomed Pharmacother 95: 914-921, 2017.

26. Lin J, Li X, Qi W, Yan Y, Chen K, Xue X, Xu X, Feng Z and Pan X: Isofraxidin inhibits interleukin-1 $\beta$ induced inflammatory response in human osteoarthritis chondrocytes. Int Immunopharmacol 64: 238-245, 2018.

27. Guo D, Cao XW, Liu JW, Niu W, Ma ZW, Lin DK, Chen JY, Lian WD, Ouyang WW and Liu J: Clinical effectiveness and micro-perfusion alteration of Jingui external lotion in patients with knee osteoarthritis: Study protocol for a randomized controlled trial. Trials 16: 124, 2015.

28. Zeng L, Xiao CZ, Deng ZT and Li RH: Chondroprotective effects and multitarget mechanisms of fu yuan capsule in a rat osteoarthritis model. Evid Based Complement Alternat Med 2017: 8985623, 2017.

29. Tong P, Xu S, Cao G, Jin W, Guo Y, Cheng Y, Jin H, Shan L and Xiao L: Chondroprotective activity of a detoxicated traditional Chinese medicine (Fuzi) of Aconitum carmichaeli Debx against severe-stage osteoarthritis model induced by mono-iodoacetate. J Ethnopharmacol 151: 740-744, 2014.

30. Cheng BC, Fu XQ, Guo H, Li T, Wu ZZ, Chan K and Yu ZL: The genus Rosa and arthritis: Overview on pharmacological perspectives. Pharmacol Res 114: 219-234, 2016. 
31. Chen GY, Tang J, Zheng P and Liu Y: CD24 and Siglec-10 selectively repress tissue damage-induced immune responses. Science 323: 1722-1725, 2009.

32. Chiba S, Baghdadi M, Akiba H, Yoshiyama H, Kinoshita I, Dosaka-Akita H, Fujioka Y, Ohba Y, Gorman JV, Colgan JD, et al: Tumor-infiltrating DCs suppress nucleic acid-mediated innate immune responses through interactions between the receptor TIM-3 and the alarmin HMGB1. Nat Immunol 13: 832-842, 2012

33. Tian X, Liu C, Shu Z and Chen G: Review: Therapeutic targeting of HMGB1 in stroke. Curr Drug Deliv 14: 785-790, 2017.

34. Rosenberg JH, Rai V, Dilisio MF and Agrawal DK: Damage-associated molecular patterns in the pathogenesis of osteoarthritis: Potentially novel therapeutic targets. Mol Cell Biochem 434: 171-179, 2017.

35. Liu L, Yang M, Kang R, Dai Y, Yu Y, Gao F, Wang H, Sun X, Li X, Li J, et al: HMGB1-DNA complex-induced autophagy limits AIM2 inflammasome activation through RAGE. Biochem Biophys Res Commun 450: 851-856, 2014.

36. Schaper F, Westra J and Bijl M: Recent developments in the role of high-mobility group box 1 in systemic lupus erythematosus. Mol Med 20: 72-79, 2014
37. Miclea RL, Siebelt M, Finos L, Goeman JJ, Löwik CW, Oostdijk W, Weinans H, Wit JM, Robanus-Maandag EC and Karperien M: Inhibition of Gsk3 $\beta$ in cartilage induces osteoarthritic features through activation of the canonical Wnt signaling pathway. Osteoarthritis Cartilage 19: 1363-1372, 2011.

38. Kim HA and Blanco FJ: Cell death and apoptosis in osteoarthritic cartilage. Curr Drug Targets 8: 333-345, 2007.

39. Yang M, Jiang L, Wang Q, Chen $\mathrm{H}$ and Xu G: Traditional Chinese medicine for knee osteoarthritis: An overview of systematic review. PLoS One 12: e0189884, 2017.

40. Tang H, He S, Zhang X, Luo S, Zhang B, Duan X, Zhang Z, Wang W, Wang Y and Sun Y: A network pharmacology approach to uncover the pharmacological mechanism of xuanhusuo powder on osteoarthritis. Evid Based Complement Alternat Med 2016: 3246946, 2016.

41. Zhou X, Lin X, Xiong Y, Jiang L, Li W, Li J and Wu L: Chondroprotective effects of palmatine on osteoarthritis in vivo and in vitro: A possible mechanism of inhibiting the Wnt $/ \beta$-catenin and Hedgehog signaling pathways. Int Immunopharmacol 34: 129-138, 2016. 